\title{
Ecoulements diphasiques dispersés : établissement des équations de bilan et problèmes de fermeture
}

\author{
J.P. Chevaillier et J. Fabre \\ Institut de mécanique des fluides de Toulouse
}

On établit les équations locales de bilan d'un écoulement diphasique dispersé isotherme, à partir de deux méthodes:

- pour la phase continue, on part de l'approche désormais classique consistant à écrire les équations phasiques instantanées; en traitant les grandeurs introduites comme des distributions, on met en évidence les termes d'interaction entre phases;

- pour la phase dispersée, les équations de bilan peuvent être développées sur la base du formalisme de la mécanique statistique; cette approche permet une meilleure prise en compte de l'évolution de la dispersion.

On compare cette méthode avec celle qui consiste à traiter la phase dispersée de la même manière que la phase continue.

\section{Introduction}

Le caractère le plus remarquable des écoulements diphasiques à deux fluides est certainement l'aptitude des interfaces à s'organiser sous l'influence des différentes forces en présence. Selon les propriétés physiques des fluides, la géométrie de l'écoulement et les conditions imposées aux limites, cette organisation peut conduire dans les situations les plus simples:

- à la séparation des phases; l'interface est alors continue et les deux domaines d'écoulement simplement connexes : c'est le cas des écoulements stratifiés lisses ou à vagues;

\begin{abstract}
- à la dispersion d'une des phases dans l'autre; les deux domaines d'écoulement sont multiplement connexes: c'est le cas des écoulements à bulles ou à gouttes.

La distinction n'est pas uniquement descriptive : elle vise à améliorer la modélisation en prenant en compte le caractère spécifique de chaque famille d'écoulement. La prédiction des écoulements repose en effet sur la résolution des équations phasiques de conservation de masse, de quantité de mouvement et d'énergie. Compte tenu du double caractère aléatoire lié à la turbulence et à la distribution spatio-temporelle des interfaces, ces équations ne sont pas fermées et le succès des modèles réside en particulier dans le choix des lois de fermeture
\end{abstract}

Dispersed diphasic flows : drawing up of balance equations and closing problems.

The local balance equations of an isotherm dispersed diphasic flow are established on the basis of two methods:

- for the continuous phase, the conventional approach is now used which consists of inscribing the instantaneous phasic equations; by treating the quantities introduced as distributions, the interaction terms between phases are revealed;

- for the dispersed phase, the balance equations may be developed on the basis of statistical mechanics formalism; this approach enables dispersion evolution to be better evaluated.

This method is compared with the one consisting of treating the dispersed phase in the same way as is the continuous phase. 
exprimant les transferts interfaciaux de masse, quantité de mouvement et énergie. On s'accorde généralement à reconnaître que la forme de ces lois dépend de la topologie des interfaces ce qui conduit à des modèles dont les fermetures sont adaptées à chaque classe d'écoulement.

On peut cependant se demander si la distinction des différentes classes d'écoulement est limitée au seul choix des lois de fermeture. En d'autres termes, existe-t-il des formulations de lois de conservation spécifiques à chaque classe d'écoulement? On se propose de donner des éléments de réponse pour le cas des écoulements isothermes finement dispersés à bulles ou à gouttes d'un mélange binaire, en comparant deux formulations différentes des équations de conservation :

- dans la première formulation, les deux phases sont traitées avec des équations identiques, en ignorant le caractère spécifique de la phase dispersée: la méthode s'appuie sur les équations locales instantanées dans chaque phase pondérées par la fonction de présence de phase;

- dans la seconde formulation la phase dispersée est traitée par une méthode largement utilisée en mécanique statistique pour traiter les ensembles de points matériels : elle permet de traduire certaines propriétés de la dispersion en faisant apparaître explicitement les distributions de taille des particules transportées; elle permet aussi de rendre compte du phénomène de nucléation pourvu qu'on sache le modéliser. Cependant la combinaison des deux méthodes nécessite une écriture claire des conditions de raccordement à l'interface.

\section{Première formulation}

\subsection{Méthode générale}

En écoulement diphasique, certaines grandeurs physiques subissent un saut à la traversée de l'interface : c'est le cas de la pression, de la masse volumique, des fractions massiques des espèces constituant le mélange... On a donc a traiter de grandeurs définies par morceaux sur chaque phase et sur l'interface. Pour écrire les équations de bilan local et exprimer en particulier les dérivées de ces grandeurs par rapport à l'espace et au temps, on utilise la notion de fonction généralisée ou distribution [1]. On obtient ainsi des équations locales instantanées pour chaque phase et pour l'interface, que l'on écrit ensuite en moyenne statistique. Cette méthode conduit à des résultats identiques à ceux obtenus par Ishii [2] en considérant les interfaces d'épaisseur finie.

En négligeant les effets irréversibles sur les interfaces, Fitremann [3] a utilisé la méthode pour l'obtention des équations de masse, quantité de mouvement, énergie et entropie. Lance $\& a l$. [4] ont dérivé les équations de transport des tensions de Reynolds et de la dissipation pour un écoulement isotherme d'un couple de fluides sans tension interfaciale.

\subsection{Equations topologiques}

Le domaine d'écoulement $\mathbf{V}$ est constitué des deux domaines $\mathbf{V}_{\mathbf{1}}$ et $\mathbf{V}_{\mathbf{2}}$ ayant ou non des propriétés de connexité, occupés par chaque fluide, dotés d'une surface de séparation $\mathbf{S}$ constituée par l'ensemble des interfaces. Les équations topologiques décrivent l'évolution dans le temps et l'espace des interfaces. Cette description est purement géométrique puisque les interfaces forment une surface non matérielle.

On définit une fonction booléenne $\chi_{k}(\mathbf{M})$, caractérisant la présence de la phase $k$, appelée fonction de présence de phase et prenant les valeurs 1 ou 0 selon que la phase est ou non présente au point $\mathbf{M}(x, t)$. Elle vérifie les propriétés :

$$
\begin{gathered}
\sum_{k=1,2} \chi_{k}=1 \\
\partial \chi_{k} / \partial t+\mathbf{U}_{i} \cdot \nabla \chi_{k}=0
\end{gathered}
$$

L'opérateur $\delta / \delta t$, équivalent à $\partial / \partial t+\mathbf{U}_{i} \cdot \nabla$, est une dérivée convective. L'équation 2 exprime que la fonction de présence ne varie pas lorsque l'on se déplace à la vitesse $\mathbf{U}_{i}$ de l'interface. On peut écrire une relation analogue pour la distribution $\delta_{i}(\mathbf{M})$, représentant la répartition de masses de Dirac sur les interfaces :

$$
\partial \delta_{i} / \partial t+\mathbf{U}_{i} \cdot \nabla \delta_{i}=0
$$

La distribution de Dirac est facilement interprétable à partir des dérivées de la fonction caractéristique :

$$
\mathbf{n}_{k} \delta_{i}=-\nabla \chi_{k}
$$

où $\mathbf{n}_{k}$ est la normale unitaire externe à la surface limitant la phase $k$.

Les équations locales instantanées $1,2,3$ et 4 peuvent être écrite en moyenne statistique, en posant :

$$
\begin{aligned}
& \alpha_{k} \triangleq \overline{\chi_{k}} \\
& \alpha_{i} \triangleq \overline{\delta_{i}}
\end{aligned}
$$

$\alpha_{k}$ et $\alpha_{i}$ représentent respectivement le taux de présence et le taux d'aire interfaciale par unité de volume [5]; on dispose donc de deux grandeurs pour caractériser la structure géométrique du milieu diphasique: en particulier, le rapport $\alpha_{k} / \alpha_{i}$ a la dimension d'une échelle de longueur et peut être interprété comme un rayon de courbure moyen des interfaces. Par cette analyse, on ne dispose donc pas d'autre information caractérisant la dispersion: en particulier le fait que les interfaces peuvent subir des évolutions par scission ou coalescence ne peut être pris en compte.

On introduit les moyennes phasiques qui sont en fait des moyennes conditionnelles prises dans chaque phase ou sur l'interface :

$$
\begin{aligned}
\alpha_{k} \bar{g}_{k}^{k} \triangleq \overline{\chi_{k} g_{k}} \\
\text { On obtient alors : } \\
\alpha_{i} \bar{g} k \triangleq \overline{\delta_{i} g_{k}}
\end{aligned}
$$

$$
\begin{gathered}
\sum_{k=1,2} \alpha_{k}=1 \\
\overline{\mathbf{n}}_{k}^{i} \alpha_{i}=-\nabla \alpha_{k}
\end{gathered}
$$

\subsection{Equations de bilan local}

Soit $g$ la densité volumique d'une grandeur transporta- 
ble, la forme générale du bilan local de $g$ est :

$$
\partial g / \partial t+\nabla \cdot(g \mathbf{U})+\nabla \cdot \Phi=\Pi
$$

où $\Phi$ est le flux diffusif et $\Pi$ le taux volumique de création-disparition de $g$. L'équation de bilan instantané s'écrit, pour un milieu diphasique, en traitant la grandeur diphasique définie partout:

$$
g \triangleq \delta_{i} g_{i}+\sum_{k=1,2} \chi_{k} g_{k}
$$

où $g_{i}$ et $g_{k}$ sont respectivement des densités surfaciques et volumiques. Après quelques manipulations algébriques, on obtient une équation diphasique instantanée que l'on décompose en deux équations phasiques et une équation interfaciale [5] :

$$
\begin{aligned}
\partial\left(\chi_{k} g_{k}\right) / \partial t+\nabla \cdot\left(\chi_{k} g_{k} U_{k}\right)+ & \nabla \cdot\left(\chi_{k} \Phi_{k}\right) \\
& -\chi_{k} \Pi_{k}+\delta_{i} \Psi_{k}=0
\end{aligned}
$$

$$
\begin{aligned}
\partial g_{i} / \partial t+\nabla_{i} \cdot\left(g_{i} \mathbf{U}_{i}\right) & +\nabla_{i} \cdot \boldsymbol{\Phi}_{i} \\
& +2 H \boldsymbol{\Phi}_{i} \cdot \mathbf{n}-\Pi_{i}-\sum_{k=1,2} \Psi_{k}=0
\end{aligned}
$$

où $\nabla_{i}$ est l'opérateur de dérivation défini par rapport aux coordonnées curvilignes de surface, $H$ la courbure moyenne de l'interface, $\Psi_{k}$ le flux interfacial de la grandeur, composé d'un terme convectif et d'un terme diffusif :

$$
\Psi_{k} \triangleq g_{k} \mathbf{n}_{k} \cdot\left(\mathbf{U}_{k}-\mathbf{U}_{i}\right)+\mathbf{n}_{k} \cdot \boldsymbol{\Phi}_{k}
$$

Les équations phasiques moyennes s'écrivent alors, en tenant compte des définitions précédentes et en faisant la décomposition en grandeurs moyennes et fluctuantes :

$$
\begin{array}{r}
\partial\left(\alpha_{k} \bar{g}_{k}^{k}\right) / \partial t+\nabla \cdot\left(\alpha_{k} \bar{g}_{k}^{k} \overline{\mathbf{U}}_{k}^{k}\right)+\nabla \cdot\left(\alpha_{k} g_{k}^{\prime} \mathbf{U}_{k}^{\prime k}\right) \\
+\nabla \cdot\left(\alpha_{k} \overline{\boldsymbol{\Phi}}_{k}^{k}\right)-\alpha_{k} \bar{\Pi}_{k}^{k}+\alpha_{i} \bar{\psi}_{k}^{i}=0 \\
\left.{\overline{g_{i} / \partial t}}^{i}+\overline{\nabla_{i} \cdot\left(g_{i} \mathbf{U}_{i}\right.}\right)^{i}+\overline{\nabla i}_{i} \cdot \boldsymbol{\Phi}_{i}^{i}+\overline{2 H} \boldsymbol{\Phi} \cdot \mathbf{n}^{i} \\
-\bar{\Pi}_{i}^{i}-\sum_{k=1,2} \bar{\psi}_{k}^{i}=0
\end{array}
$$

On retrouve les équations de bilan de la masse totale, de la quantité de mouvement et de la masse d'une des deux espèces du mélange binaire, supposé non réactif à partir du tableau suivant :

$\begin{array}{lcccccc} & g_{k} & g_{i} & \boldsymbol{\Phi}_{k} & \boldsymbol{\Phi}_{i} & \Pi_{k} & \Pi_{i} \\ \text { masse totale } & \rho_{k} & 0 & 0 & 0 & 0 & 0 \\ \text { quantité de mouv. } & \rho_{k} \mathbf{U}_{k} & 0 & p_{k} \mathbf{I}-\mathbf{\Sigma}_{k}-\sigma \mathbf{I} & \rho_{k} \mathbf{g} & 0 \\ \text { masse d'espèce } & \rho_{k} c_{k} & 0 & J_{k} & 0 & 0 & 0\end{array}$

La résolution du système d'équations obtenu nécessite la détermination des variables principales de l'écoulement, notamment de $\alpha_{k}, \bar{g}_{k}^{k}, \overline{\mathrm{U}}_{k}^{k}$. Or certains termes de (16) et (17) sont a priori inconnus et doivent être fermés par des lois physiques: on trouvera une discussion des fermetures d'équations formulées de manière analogue, dans l'article de Drew [6]. Il faut noter que le succès de la modélisation repose en grande partie sur l'aptitude des lois à bien prendre en compte le transport phasique turbulent ( $3^{\mathrm{e}}$ terme de l'éq. 16$)$, et le transfert entre phases : $\alpha_{i} \bar{\psi}_{k}^{i}$. Du point de vue physique, le flux surfacique interfacial $\bar{\psi}_{k}^{i}$ doit dépendre de la taille des inclu- sions et donc de leur distribution statistique. Or le modèle est limité à la prise en compte de la seule échelle de longueur de la dispersion $\alpha_{k} / \alpha_{i}$ : on peut donc envisager de fermer par une loi de la forme $\bar{\psi}_{k}^{i}\left(\alpha_{k} / \alpha_{i}\right)$. Mais, comme il manque une équation de transport de l'aire interfaciale, il n'est pas possible de prédire l'évolution de $\alpha_{i}$. C'est certainement l'une des lacunes de cette formulation.

\section{Deuxième formulation}

\subsection{Equation de bilan de la densité de proba- bilité}

La phase dispersée est supposée constituée de particules homogènes et sphériques, hypothèse acceptable pour les fines dispersions. A un instant $t$, chacune d'elles est définie par sa position $\mathbf{x}$, sa vitesse $\mathbf{u}$ et son rayon $r$. Par interaction avec le champ aléatoire de vitesse et de pression de la phase continue, les coordonnées de phase $(\mathbf{x}, \mathbf{u}, r)$ de chaque particule varient au cours du temps de manière aléatoire.

Pour définir la topologie de la phase dispersée, on considère un ensemble de réalisations indépendantes du même écoulement. On peut ainsi associer une densité de répartition numérique $f(\mathbf{x}, \mathbf{u}, r ; t)$, définie dans l'espace des phases en chaque point de coordonnées, représentant la probabilité d'avoir une particule dans le domaine $[\mathbf{x}-\mathbf{x}+\mathbf{d x} ; \mathbf{u}-\mathbf{u}+\mathbf{d u} ; r-r+d r]$. En établissant le bilan numérique des particules dans l'espace des phases, on obtient après quelques calculs, l'équation fondamentale d'évolution de la population de particules.

C'est une équation de Liouville généralisée :

$\partial f / \partial t+\nabla_{x} \cdot(f \mathbf{u})+\nabla_{\mathbf{u}} \cdot(f \dot{\mathbf{u}})+\partial(f \dot{r}) / \partial r=\omega$

où la notation ( ${ }^{\circ}$ représente la dérivée particulaire $d / d t$, et où $\omega$ est le taux moyen de création ou disparition de particules par scission ou coalescence. On peut noter que le phénomène de nucléation et celui de collapse de particules ne sont pas forcément contenus dans $\omega$, puisqu'on peut considérer dans la distribution numérique, des particules de très faible rayon qui sont les germes de la nucléation ou les résidus du collapse.

L'équation (18) est un bilan dans l'espace des phases. Elle n'est parfaitement définie que si l'on connaît :

- les lois donnant les vitesses des coordonnées de phase : u, $\dot{\mathrm{u}}, \dot{r}$;

- le terme source $\omega$.

Le cas du terme source sera discuté plus loin. Le problème de l'obtention des lois donnant les vitesses de phase est complexe puisqu'il suppose la possibilité de modéliser, pour les configurations possibles du système diphasique, l'interaction entre les particules et l'écoulement. En fait on retrouve un problème analogue à celui de la première formulation. Achard [7] a discuté les méthodes d'obtention de ces lois ainsi que les modèles d'interactions statistiques. 


\subsection{Moyennes numériques et phasiques}

Le nombre moyen de particules dans un volume unitaire du milieu diphasique est donné par :

$n(\mathbf{x}, \mathbf{t}) \triangleq \int f d \mathbf{u} d r$ (pour l'ensemble de la population)

$n_{r}(x, r, t) \triangleq \int f d \mathbf{u}$ (pour chaque classe de rayon) (20)

Pour une grandeur transportable $\gamma$ attachée à la phase dispersée, la moyenne numérique par classe de rayon est définie par:

$$
\langle\gamma\rangle \triangleq \int \gamma f d \mathbf{u} / n_{r}
$$

Ces définitions reposent sur la représentation statistique de la phase dispersée que l'on introduit. On va montrer cependant que cette représentation particulière est cohérente avec la représentation par les moyennes phasiques introduites dans la première formulation.

En effet, $v$ étant le volume d'une particule, on peut exprimer le taux de présence $\alpha_{d}$ de la phase dispersée dans le milieu par:

$$
\alpha_{d} \triangleq \int v f d \mathbf{u} d r
$$

ainsi que la moyenne $\bar{g}^{d}$ d'une grandeur volumique $g(g \triangleq \gamma / v)$ :

$$
\alpha_{d} \overline{\boldsymbol{g}}^{d} \triangleq \int \boldsymbol{g} v f d \mathbf{u} d r
$$

Le taux d'aire interfaciale $\alpha_{i}$ s'écrit aussi pour des particules sphériques de surface unitaire $s$ :

$$
\alpha_{i} \triangleq \int s f d \mathbf{u} d r=3 / 2 \alpha_{d} \bar{H}^{d}
$$

où la courbure moyenne $\bar{H}^{d}$ est définie par :

$$
\alpha_{d} \bar{H}^{d} \triangleq \int 2 r^{-1} \text { vf } d \mathbf{u} d r
$$

La moyenne de $g$ sur l'interface peut être définie de manière analogue :

$$
\alpha_{i} \bar{g}^{i} \triangleq \int g_{i} s f d \mathbf{u} d r
$$

Les moyennes $\alpha_{d}, \alpha_{i}, \bar{g}^{d}, \bar{g}^{i}$ s'identifient avec celles définies dans la première formulation.

\subsection{Equations de bilan de la phase dispersée par classes de rayon}

En multipliant l'équation de bilan 18 par $\gamma$, puis en intégrant dans l'espace des vitesses, on obtient:

$$
\begin{aligned}
\partial\left(n_{r}\langle\gamma\rangle\right) / \partial t+\nabla \cdot & \left(n_{r}\langle\gamma \boldsymbol{u}\rangle\right)+ \\
& \partial\left(n_{r}\langle\gamma \dot{r}\rangle\right) / \partial r=n_{r}(\langle\Gamma\rangle+\langle\dot{\gamma}\rangle)
\end{aligned}
$$

où $\Gamma$ est le taux relatif de création de la grandeur $\gamma$ associé à la création de particules, défini par:

$$
\Gamma \triangleq \gamma \omega / f
$$

En faisant $\gamma \equiv 1$, on obtient le bilan numérique par classe de rayon:

$\partial n_{r} / \partial t+\nabla \cdot\left(n_{r}\langle\mathbf{u}\rangle\right)+\partial\left(n_{r}\langle\dot{r}\rangle\right) / \partial r=n_{r}\left\langle\Gamma_{n}\right\rangle$

$$
\Gamma_{n} \triangleq \omega / f
$$

L'équation 29 est souvent proposée en association avec les équations phasiques pour déterminer les caractéristiques géométriques de la phase dispersée (Greenspan \& Ungarish [8]). Elle est fondamentale lorsque le transfert de masse à l'interface provoque une modification notable de la granulométrie.

En combinant 27 et 29 et en faisant la décomposition classique en moyenne et fluctuation $\left(\gamma^{0} \triangleq \gamma-\langle\gamma\rangle\right)$, on obtient :

$$
\begin{aligned}
& \frac{\partial\langle\gamma\rangle / \partial t}{1}+\underline{\langle\mathbf{U}\rangle \cdot \nabla\langle\gamma\rangle}+\underline{\langle\dot{r}\rangle \partial\langle\gamma\rangle / \partial r} \\
& +\underline{n_{r}^{-1}\left\{\nabla \cdot\left(n_{r}\left\langle\gamma^{o} \mathbf{u}^{o}\right\rangle\right)+\partial\left(n_{r}\left\langle\gamma^{o} \dot{r}^{o}\right\rangle\right) / \partial r\right\}}= \\
& \underline{\langle\Gamma\rangle+\langle\dot{\gamma}\rangle}-\overline{\langle\gamma\rangle\left\langle\Gamma_{n}\right\rangle}
\end{aligned}
$$

Les différents termes de l'équation 31 représentent:

1) la variation temporelle et la convection par le mouvement moyen;

2) la redistribution dans les classes de rayon par croissance-décroissance des particules;

3) le transport et la redistribution par diffusion turbulente;

4) la variation de la grandeur due à la création-disparition des particules.

L'équation 31 permet donc de représenter l'évolution de la granulométrie d'une population de particules en interaction avec le milieu.

\subsection{Equations de bilan moyen de la phase dispersée}

On peut tout aussi bien rechercher uniquement une information plus grossière en effectuant des moyennes sur l'ensemble de la dispersion. On obtient l'équation générale en multipliant l'équation 18 par $g v$, et en intégrant sur l'espace des vitesses et des rayons:

$\partial\left(\alpha_{d} \bar{g}^{d}\right)+\nabla \cdot\left(\alpha_{d} \overline{g u}^{d}\right)=\alpha_{d} \bar{G}^{d}+{\overline{\alpha_{d}(\dot{g} v) / v}}^{d}$

Le dernier terme représente la contribution du transfert interfacial; il peut être développé selon :

$$
\alpha_{d} \overline{(\dot{g} v) / v}^{d}=\alpha_{d} \overline{\dot{g}}^{d}+\alpha_{i} \overline{g r}^{d}
$$

En faisant la décomposition habituelle:

$$
g^{\prime} \triangleq g-\bar{g}^{d} \quad \mathbf{u}^{\prime} \triangleq \mathbf{u}-\overline{\mathbf{u}}^{d}
$$

et en utilisant le bilan volumique, on obtient :

$$
\begin{aligned}
\frac{\partial \bar{g}^{d} / \partial t}{}+ & +\overline{\mathbf{u}}^{d} \cdot \nabla \bar{g}^{d}+\frac{\alpha^{-1} \nabla \cdot\left(\alpha_{d} \overline{g^{\prime} \mathbf{u}^{\prime}}\right)}{2} \\
= & \left.=\bar{G}^{d}-\bar{g}^{d} \bar{G}_{\alpha}^{d}+\overline{\bar{g}^{d}+\alpha_{i} \alpha_{\bar{d}}^{-1}\left(\overline{g \dot{r}}^{d}\right.}-\bar{g}^{d} \bar{r}^{d}\right)
\end{aligned}
$$

où $G_{\alpha}$ est le taux de création-disparition de $\alpha_{d}$ dans le processus de scission-coalescence.

Dans cette dernière équation les différents termes représentent :

1) la variation temporelle et le transport par le mouvement moyen; 
2) le transport par diffusion turbulente;

3) la création et disparition par variation du nombre de particules;

4) la variation par transfert interfacial et croissance des particules.

\section{Comparaison des deux formulations et conclusions}

\subsection{Remarques préliminaires}

Telles qu'elles sont écrites les équations de bilan correspondant aux deux formulations ne contiennent pas la même information.

En effet dans la première méthode, on part des bilans dans chaque phase et on applique en dernier lieu le traitement statistique; les équations obtenues contiennent donc la physique, à l'exemple de l'équation de quantité de mouvement qui exprime la loi fondamentale de la mécanique.

La seconde méthode n'est en somme qu'un traitement statistique de la dispersion selon la position, la vitesse et la taille; à ce stade de l'écriture, la physique n'est pas réellement incluse, puisqu'à titre d'exemple, aucun bilan de forces n'a été écrit pour exprimer la conservation de la quantité de mouvement.

\subsection{Comparaison des formulations}

On se limitera à l'écriture du bilan de masse qui est suffisamment significatif pour la comparaison. On considère de plus le cas d'un écoulement isovolume dans chaque phase, de sorte que la conservation de la masse se traduira par un bilan de volume.

Dans le cas de la première formulation $(\S 2.3)$, les équations pour chaque phase s'écrivent à partir de (15) et (16) :

$$
\begin{gathered}
\partial \alpha_{k} / \partial t+\nabla \cdot\left(\alpha_{k} \overline{\mathbf{U}}_{k}^{k}\right)+\alpha_{i} \overline{\mathbf{n}_{k} \cdot\left(\mathbf{U}_{k}-\mathbf{U}_{i}\right)}{ }^{i}=0 \\
\sum_{k=1,2} \rho_{k} \overline{\mathbf{n}_{k} \cdot\left(\mathbf{U}_{k}-\mathbf{U}_{i}\right)^{i}}=0
\end{gathered}
$$

On ne peut espérer calculer l'évolution de $\alpha_{k}$ que si l'on se donne une loi de fermeture sur le flux de masse interfacial $\rho_{k} \overline{\mathbf{n}_{k} \cdot\left(\mathbf{U}_{k}-\mathbf{U}_{i}\right)^{i}}$, et sur le taux d'aire interfaciale $\alpha_{i}$.

Le flux de masse à l'interface est fonction du déséquilibre thermodynamique local et d'une vitesse de transfert, fonction du glissement moyen entre phases (cf. par ex. Ranz \& Marshall [9]) et des échelles de vitesse de la turbulence. Il dépend indirectement de l'échelle de longueur de la dispersion, c'est-à-dire de $\alpha_{k} / \alpha_{i}$. En outre le flux volumique est directement proportionnel à $\alpha_{i}$. On voit donc tout l'intérêt qu'il y a à prédire le taux d'aire interfaciale pour lequel on ne dispose pas d'équation de transport. Or son taux de variation est particulièrement important dans la nucléation et le collapse. Cette formulation présente donc une lacune importante.

En utilisant des équations de bilan moyen de la seconde formulation $(\S 3.4)$, on peut écrire un bilan de volume et un bilan sur le nombre pour la phase dispersée :

$$
\begin{gathered}
\partial \alpha_{d} / \partial t+\nabla \cdot\left(\alpha_{d} \overline{\mathbf{u}}^{d}\right)=\alpha_{i} \bar{r}^{d} \\
\partial n / \partial t+\nabla \cdot\left(n \overline{\mathbf{u}}^{d}\right)=N
\end{gathered}
$$

Par identification avec (36) et (37), il est clair que :

$$
\left.m \triangleq \rho_{d} \bar{r}^{d}=\rho_{c} \overline{\mathbf{n}_{c} \cdot\left(\mathbf{U}_{c}-\mathbf{U}_{i}\right.}\right)^{i}
$$

On peut donc associer à (38) et (39) l'équation 36 pour traiter la phase continue. La fermeture des flux de masse à l'interface se pose donc de la même manière. On dispose cependant d'une équation supplémentaire sur la population des particules que l'on doit fermer en incluant les processus de scission et de coalescence, fixant le taux de création-disparition $N$. Cependant il n'y a pas de moyen de prédire $\alpha_{i}$ à partir de (39).

On est donc amené à utiliser les moyennes par classe de rayon ( $\$ 3.3)$, c'est-à-dire à résoudre l'équation 29 en se donnant la fermeture pour $\left\langle\Gamma_{n}\right\rangle$. En écoulements à bulles, les modèles de Hinze [10] pour la scission et de Thomas [11] pour la coalescence, peuvent être utilisés; ils prédisent que la scission résulte de l'instabilité des bulles de rayon supérieur à $r_{1}$ et que la coalescence est impossible à moins que leur rayon soit inférieur à $r_{2}$ :

$$
\begin{gathered}
2 r_{1} \sim\left(\sigma / \rho_{c}\right)^{3 / 5} \varepsilon^{-2 / 5} \\
2 r_{2} \sim 2,4\left(\sigma^{2} h^{2} / \mu_{c} \rho_{c} \varepsilon\right)^{1 / 4}
\end{gathered}
$$

où $\sigma$ est la tension interfaciale, $h$ l'épaisseur critique de rupture du film entre bulles, $\mu$ la viscosité dynamique et $\varepsilon$ la dissipation turbulente.

Les phénomènes de nucléation et collapse interviennent dans la classe des très petits rayons. Pour que la modélisation les prennent en compte il faut donc que la distribution $n_{r}(r)$, ait une valeur finie au voisinage de $r=0$.

A partir de la distribution par classe de rayon, on peut déterminer le taux de présence, le taux d'aire interfaciale et le flux massique volumique :

$$
\begin{gathered}
\alpha_{d}=\int v n_{r} d r \\
\alpha_{i}=\int s n_{r} d r \\
\alpha_{i} m=\alpha_{i} \rho_{d} \overline{\dot{r}_{d}}=\rho_{d} \int \dot{v} n_{r} d r
\end{gathered}
$$

La loi de croissance des particules $\dot{v}$ peut être écrite par classe de rayon.

On dispose d'un ensemble de relations permettant de traiter l'évolution d'une population de particules en prenant en compte le transfert de masse, ainsi que la coalescence et la scission.

Adresse des auteurs

Messieurs J.P. Chevaillier

Institut de mécanique des fluides de Toulouse

Allée du Professeur Camille Soula

31400 Toulouse

Tél. : 61.28 .58 .03 


\section{Bibliographie}

[1] Schwartz L. - Théorie des distributions, Hermann Paris, 1966.

[2] IsHII M. - Thermo-fluid dynamic theory of two-phase flow, Eyrolles Paris, 1975.

[3] Fitremann J.M. - Ecoulements diphasiques: théorie et application à l'étude de quelques régimes d'écoulements verticaux ascendants d'un mélange gaz-liquide, Thèse, Université Pierre et Marie Curie, Paris, 1977.

[4] Lance M., Marie J.L., Charnay G., Bataille J. - Les équations de la turbulence dans un écoulement diphasique incompressible en l'absence de transfert de masse, $C . R$. Acad. Sc., Paris, 288, A, 957-960.

[5] FABRE J. - Ecoulements diphasiques, cours de Dea de l'ENSEEIHT, Toulouse, 1982.
[6] DREw D.A. - Mathematical modeling of two-phase flow, Ann. Rev. Fluid Mech., 15, 261-291, 1983.

[7] AChard J.L. - Contribution à l'étude théorique des écoulements diphasiques en régime transitoire, Thèse, Institut National Polytechnique de Grenoble, 1978.

[8] Greenspan H.P., UNGarish M. - On hindered settling of particles of different sizes, Int. J. Multiphase Flow, 8, 6, 587-604, 1982.

[9] RANZ W.E., MARShall W.R. - Evaporation from drops, Chemical Engineering Progress, Part I, 48, 3, 141-146, Part II, 48, 4, 173-180, 1952.

[10] HiNZE J.O. - Fundamentals of the hydrodynamic mechanism of splitting in dispersion process, AIChE J., 289-295, 1955.

[11] Tномаs R.M. - Bubble coalescence in turbulent flow, Int. J. Multiphase Flow, 7, 6, 709-717, 1981.

\section{Discussion}

Président :

Le Président. - Je remercie M. CHEVAILLIER. Il est particulièrement délicat d'exposer un modèle qui se traduit par une masse d'équations. $\mathrm{Y}$ a-t-il des questions ?

M. DELHAYE. - J'ai beaucoup apprécié la clarté de cet exposé. Il y a une dizaine d'années, nous avions travaillé à Grenoble avec $M$. ACHARD sur le même problème, avec une méthode analogue. Je voudrais savoir s'il y a une originalité par rapport à l'approche de $M$. ACHARD?

M. CheVAalllier. - M. ACHARD a, en effet, développé la méthode générale d'obtention des équations par le formalisme statistique classique utilisé en mécanique des particules; il a ensuite focalisé son attention sur les équations de transport des moments et tenté de l'appliquer au cas d'un écoulement en tube. Dans notre cas, on s'est attaché au cas des écoulements 3D et on s'est intéressé aux équations de bilan par classe de rayons et non aux équations des moments où l'information est dégradée.

Le Président. - C'est un cas particulier du modèle de $M$. ACHARD que vous appliquez à des écoulements isothermes à petites bulles.

M. ChevallLIER. - Les équations de départ sont effectivement identiques : ce sont en fait les équations de LiouvilleBoltzmann. Nous avons essayé de montrer l'intérêt de traiter les équations par classe de rayons qui permettent de rendre compte de l'évolution d'une population de particules.

Le Président. - Y a-t-il et envisagez-vous des comparaisons avec des résultats expérimentaux?

M. CHEVAILLIER. - A ce stade, ce n'était pas notre objectif.

Le Président. - Mais vous comptez le faire?

M. CHEVAILLIER. - Le modèle statistique ne peut fournir aucun résultat tant qu'il ne contient pas les fermetures appro. priées concernant $\mathrm{u}, \dot{\mathrm{u}}, \dot{r}$. Il ne fournit que le cadre théorique pour traiter le problème de l'évolution d'une population. Les différents exposé de cette session montrent la nécessité d'une modélisation plus fine des problèmes d'évolution des populations lorsque le transfert de masse intervient.

Le Président. - Le problème que vous cherchez à traiter. finalement, c'est l'évolution d'un écoulement à bulles. Le problème de la nucléation est en amont.

M. CHEVAILLIER. - Nous n'avons pas étudié la nucléation.

M. DELHAYE. - Je pense que ce sont des méthodes tout à fait intéressantes. Encore faudrait-il aller plus loin, c'est-à-dire confronter la théorie avec l'expérience.

M. CHEVAILLIER. - Nous sommes d'accord.

M. VERNIER. - Pourriez-vous nous expliquer pourquoi n'y a-t-il pas d'équation de transport pour la densité interfaciale, ce que j'ai appelé ce matin $\ell \bar{S}^{1}$ ?

M. ChEVAlLLIER. - Cette équation de transport n'est pas écrite pour le moment, elle n'existe pas dans la littérature; tout au moins, je ne l'ai pas vue.

M. VERNIER. - La moyenne statistique de l'Eq. (3) pourrait fournir l'équation de $\alpha_{i}$.

M. CHEVAlLLIER. - C'est parfaitement exact. Il faut cependant que l'équation obtenue ne comporte pas que des termes à modéliser, sinon, autant adopter une démarche totalement heuristique.

M. THIRRIOT. - A propos des bilans d'énergie, peut-on avoir une idée sur la part d'énergie mise en jeu dans le développement des surfaces par rapport aux autres parts du bilan?

M. CHEVAILLIER - On peut donner un exemple: dans le processus de scission des bulles par la turbulence, on peut estimer que le rayon de celles-ci s'ajoute de telle sorte que l'énergie interfaciale est du même ordre de grandeur que l'énergie turbulente.

Le Président. - Nous allons arrêter cette discussion et passer au dernier exposé, qui sera présenté par M. MIDOUX. On va voir un très grand nombre de cas possibles et un peu moins d'équations. 